\title{
On the photometric behaviour of the Pleiades K dwarf HII 1883 ${ }^{\star}$
}

\author{
D. Clarke
}

\begin{abstract}
Department of Physics and Astronomy, The University, Glasgow G12 8QQ, Scotland, UK
\end{abstract}
Received 11 September 2003 / Accepted 17 March 2004

\begin{abstract}
Photometric data from a variety of sources have been analysed to check if effects of differential rotation or evolutionary spin-down can be detected in HII 1883. Although the same light-curve form may be maintained for several years, shape changes and phase shifts do not allow sufficiently long time-bases for the required accuracy of period determination. One form of light-curve may have a tendency to recur with phase coherence. Using this observation allows determination of three potentially more accurate periods relative to the one previously established, but the choice of any revised value is subject to imprecise knowledge of the integer number of cycles over the time-base. The phase-locked tendency, if confirmed, requires some mechanism for spots to recur at a given longitude. A suggestion of the star being a magnetic oblique rotator is mooted but other observational and theoretical support would be required to promote this notion further. Evidence of small short-term changes occurring over just a few periods is explored. Comparison of the contemporaneous light-curve with the $\mathrm{H} \alpha$ line emission RV variations reveals a phase difference of $90^{\circ}$, so making a connection that the disturbances producing the photometric signals have the same location as those generating at least some part of the $\mathrm{H} \alpha$ emission. Finally a multi-spot model is presented to mimic the observed light-curves.
\end{abstract}

Key words. stars: solar-type - stars: rotation

\section{Introduction}

Evidence that $\mathrm{G}$ and $\mathrm{K}$ dwarfs in the Pleiades exhibit shortperiod photometric variability was reported by Alphenaar \& van Leeuwen (1981) and Meys et al. (1982). These early results were summarised in van Leeuwen \& Alphenaar (1982) and were later discussed more comprehensively, in van Leeuwen et al. (1987, vLAM). The first light-curve of the K dwarf HII 1883 was obtained by van Leeuwen \& Alphenar (1982), the determined period being $\sim 0.2354$, revealing the star's rapid rotation. In fact, this star displays the highest $v \sin i$ $\left(\sim 140 \mathrm{~km} \mathrm{~s}^{-1}\right.$ - see Jones et al. 1996) of its type monitored in the Pleiades. Phased $V$-band measurements of Oct. 1980 are displayed in Fig. 1. The underlying light-curve reveals a brightness drop covering a phase interval $\sim 0.4$, with the recovery to maximum light occupying the remaining part of the cycle; the amplitude of the variation is $\sim 0$. 1 . This near sinusoidal light-curve was confirmed by further measurements of Stauffer (1984).

Following the discovery of the variability of HII 1883 , Stauffer et al. (1986, SDA) showed that the light-curve maintained its shape for over four years before breaking down, i.e., after a few thousand stellar revolutions. The disposition pattern of the disturbances or spots is remarkably stable for long intervals of time, in contrast with the Sun whose individual spots barely persist for longer than a single rotation cycle. All these studies were undertaken using photoelectric instruments

\footnotetext{
* e-mail:d.clarke@astro.gla.ac.uk
}

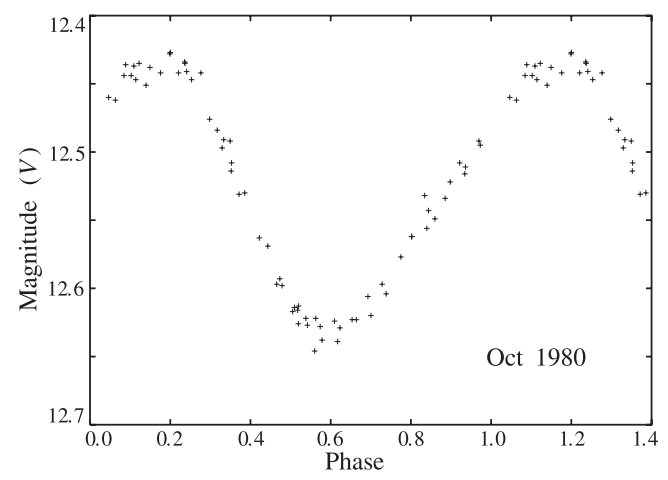

Fig. 1. The $V$-band light-curve for HII 1883 constructed from the Oct. 1980 data of vLAM based on the ephemeris of $\mathrm{HJD}=2444500.0+0.23540$

but recently photometry of HII 1883 have been extended by Krishnamurthi et al. (1998) using CCD imaging techniques.

Evidence supporting the notion that the periodicity relates directly to rotation is provided by colour photometry. The brightness modulation is engendered by localised disturbances with temperatures different from those of the regular photosphere or chromosphere. Similar in-phase light-curves are therefore obtained using different passbands, with the amplitude decreasing as the selected effective wavelength runs from blue to red. The correlation between the $B$ and $V$ light-curves of HII 1883 over the whole cycle is demonstrated by plotting simultaneous measurements for the two colours, as in Fig. 2. 


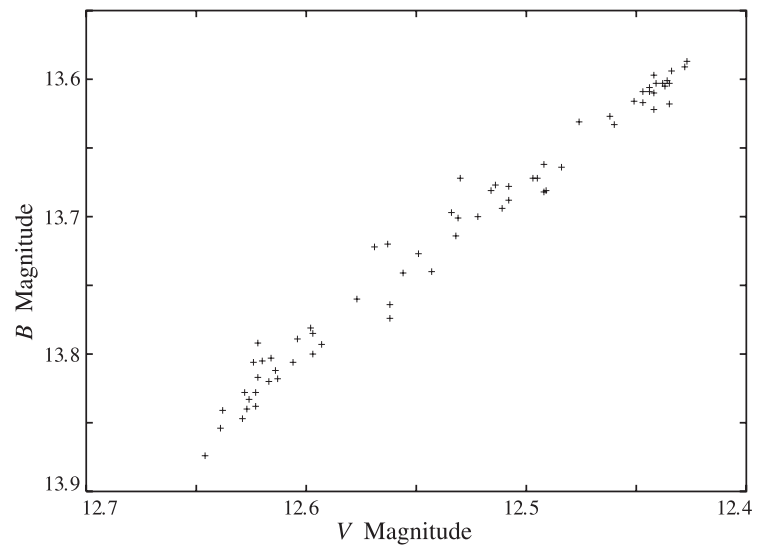

Fig. 2. The $B$ and $V$ magnitudes of HII 1883 obtained by vLAM in Oct. 1980 display a high degree of correlation in the two bands over the complete cycle from minimum to maximum light.

It may be noted that the gradient of the near-linear relationship is not unity but 1.21 , indicating a larger amplitude variation in $B$ relative to $V$, with the disturbances displaying temperature differences of a few hundred degrees relative to the pristine stellar surface. Simple photometry alone, however, does not allow distinction to be made as to the features being dark or bright. If they are dark, minimum light phase corresponds to the presentation of the most disturbed hemisphere, with maximum light relating to the projection of the quieter hemisphere; if the features are bright zones, the opposite conclusion simply applies as to which light-curve extreme corresponds to the presentation of the most affected hemisphere.

The literature provides a plethora of papers describing observations and models for spot distributions of rotationally modulated stars, commonly referred to as BY Dra variables. These include the study by Stout-Batalha \& Vogt (1999) of the Pleiades stars HII 686 and HII 3163. Insight on the BY Dra syndrome has also been discussed by Young et al. (1990) in respect of simultaneous photometry and high resolution spectrometry of the rapidly rotating $\mathrm{M}$ dwarf, GL 890; Strassmeier et al. (1993) have studied the rapidly rotating K2 dwarf LQHya, thought to be of similar age to the Pleiades, and have compared their Doppler images of spot distribution with the photometric behaviour. Because of its high apparent brightness, AB Dor has been the subject of intensive observational studies, this star being part of the Pleiades moving group (see Innes et al. 1986). Other relevant works are by Kürster et al. (1994), Hussain et al. (1997) and Donati \& Collier Cameron (1997). As for the morphologies of the light-curves, most provide nearsinusoidal shapes (e.g., see Fig. 3 in Barnes et al. 1999). The stars monitored by vLAM all had variability well described by a fundamental frequency (first harmonic) and its second harmonic, their combination being used to determine the rotational periods. By applying Fourier analysis to a simple model involving spots traversing the apparent disk and being occulted during half of the rotational cycle, Clarke (2003) has demonstrated why this curve fitting strategy is effective.

The thrust of the study here is the analysis of photometric data of HII 1883 obtained in various seasons over a 20 year interval. One aim is to explore the possible detection of differential rotation by spot migration affecting changes of the apparent stellar period linked to waveform changes as undertaken by Vogt (1981) for the archetype star, BY Dra. Also, as the time baseline increases, there is a possibility of detecting evolutionary spin-down of the star, predicted by models such as by Endal \& Sofia (1981). An approximate value of the spin-down rate for HII 1883 may be obtained by comparing its current period $(\sim 0.25)$ with the shortest period stars of similar spectral type in a cluster of known older age. For example, according to Radick et al. (1987), the fastest noted rotation period for $\mathrm{K}$ dwarfs in the Hyades with age $\sim 600 \mathrm{Myr}$ is 9 days. Taking the age of the Pleiades as $\sim 100 \mathrm{Myr}$, the spin-down rate, based on a linear decay, is $8.75 / 500 \times 10^{6}$ days per year. This translates to a change in period of $\sim 0.000000175$ per 10 years or, as based on the current period, one part in $1.43 \times 10^{6}$ per 10 years. In the earlier evolutionary stages, the spin-down rate is likely to be greater than this, and changes of the order of one part in $10^{6}$ per 10 years might be expected. According to the study by SDA, the period of HII 1883 was determined to just better than 1 part in $10^{5}$ with measurements using a time baseline of $\sim 3$ years. With further accurate period determinations and a baseline of several tens of years, detection of spin-down in HII 1883 is a realistic challenge.

Tentative links are also made between the light-curves and variations in the emission of $\mathrm{H} \alpha$ as measured by Marcy et al. (1985). Finally a simple model is presented demonstrating how light-curves can be mimicked by many fragmented features around the stellar surface.

\section{Period analyses}

The photometric data used in the analyses are taken from vLAM (Table 4) covering Oct./Nov. 1980 and Oct. 1981, SDA (Tables I, IV, VI and V) covering Jan. 1983 to Nov. 1985 and selected unpublished data (DB1-6) provided by Dr Dana Backman, these allowing reasonable period determinations for 1995 to 1999. A small data set for March 2001 was also available but this did not provide a good value for the stellar period.

To determine the periodicity associated with each data set, the Lafler-Kinman (LK) statistic, as described by Clarke (2002), was applied to each observational run in isolation. Periodograms were generated with identical grid spacing and were considered with the same criteria to obtain error estimates of the determined period, irrespective of the time span of the measurements, their number and their quality. The determined period values are listed in Table 1 and their time progression is displayed in Fig. 3. It is obvious that no change of period is detectable over the interval of 20 years. The intervals between 1980-1985 and 1995-1999 provided weighted mean periods of $0.23530 \pm 0.00006$ and $0.23528 \pm$ 0.00008 respectively. Ignoring the two poorly determined values, one in each of these intervals, the overall weighted mean is $0.23529 \pm 0.00005$.

It may be noted that these values are close to those promoted by VLAM and SDA, but are significantly smaller. The period suggested by SDA was developed by taking the 
Table 1. The determined periods of HII 1883 for the 12 data sets over the 20 year period from Oct./Nov. 1980 to Oct. 1999 are listed with their associated uncertainties. Column 1 provides an approximate HJD for the mid-point of each data set and Col. 4 lists the source of the material, the bracketed numerals referring to the particular table within the referenced paper.

\begin{tabular}{cccl}
\hline \hline $\begin{array}{c}\text { HJD } \\
+2400000\end{array}$ & $\begin{array}{c}\text { Period } \\
\text { (days) }\end{array}$ & $\begin{array}{c}\text { Uncertainty } \\
\text { (days) }\end{array}$ & \multicolumn{1}{c}{ Source } \\
\hline 44555 & 0.23524 & \pm 0.00009 & vLAM O/N 1980 \\
44905 & 0.23538 & \pm 0.00014 & vLAM Oct. 1981 \\
45360 & 0.23545 & \pm 0.00018 & SDA(I) Jan. 1983 \\
45674 & 0.23504 & \pm 0.00059 & SDA(IV) Dec. 1983 \\
46002 & 0.23532 & \pm 0.00033 & SDA(VI) Oct. 1984 \\
46377 & 0.23526 & \pm 0.00022 & SDA(V) Nov. 1985 \\
50005 & 0.23521 & \pm 0.00010 & DB1 Oct. 1995 \\
50363 & 0.23542 & \pm 0.00025 & DB2 Oct. 1996 \\
50457 & 0.23542 & \pm 0.00025 & DB3 Jan. 1997 \\
50733 & 0.23547 & \pm 0.00040 & DB4 Oct. 1997 \\
51097 & 0.23538 & \pm 0.00017 & DB5 Oct. 1998 \\
51461 & 0.23545 & \pm 0.00041 & DB6 Oct. 1999 \\
\hline
\end{tabular}

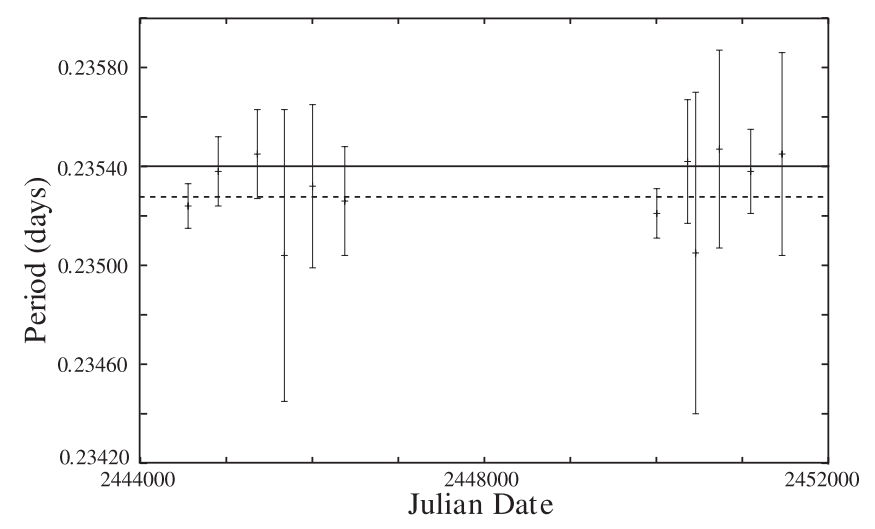

Fig. 3. The periods of HII 1883 with $1 \sigma$ uncertainties as listed in Table 1 are plotted against a mean HJD of each data set. The strong horizontal line corresponds to a period of 0.23540 while the dashed line indicates the weighted mean $(0.23529)$ of the plotted measurements.

preliminary value of Meys et al. (1982) and adjusting it so that the phase of the light-curve was maintained for the new photometry of early 1983 (see SDA, Table 1). By extending the time baseline and applying the phase preservation method, an excellent strategy when data sets of mixed type are considered, SDA suggested a value of 0.25340 . Measurements made at the end of 1983 [SDA(IV)] showed, however, that if this period were stable, the phase of the variations must have changed significantly during 1983.

According to the curve fitting procedure used by vLAM, the periods obtained from their two observing seasons were $0.23538 \pm 0.00006$ and $0.23540 \pm 0.00007$ respectively. Application of either of these values gives no significant drift in phase of the waveforms between 1980 and 1981, whereas the periods determined by the Lafler-Kinman statistic do exhibit a substantial phase difference between the two seasons. It may well be that the different approaches of curve fitting and the LK statistic provide systematic differences in their results. For example, the lower values from the LK method may result from the investigated waveform not being exactly sinusoidal. However, this does not affect the conclusion that the measured periods of HII 1883 at particular seasons have not been detected as being variable over a time base of some 20 years. Possible spot migrations to latitudes away from the equator, with associated differential rotation affecting the photometric modulation, have not been detected by simple period determinations. Any evidence of evolutionary spin-down is also absent.

The light-curve forms for any season may be simply obtained by phase binning the data based on the SDA period using an ephemeris of $\mathrm{HJD}=2444500.0+0.23540$. Figure 4 displays the underlying forms of the light-curves for 12 seasons. Some of the original data were tabulated as differential values (HII 1883 - reference star) and the plotted magnitudes have therefore been adjusted with arbitrary offsets so that the values lie in the range of the original vLAM light-curves. It is consequently inappropriate to compare the mean light levels from season to season. It should be noted that the effective wavelengths of the passbands may not be the same for the various data sets as the measurements were made using several different instruments. For example, $V$-band measurements are available in VLAM but the tabulated values of SDA(I) are from unfiltered differential photometry. Hence, again, direct comparisons of the light-curve amplitudes are not exactly meaningful.

As noted in previous studies, the form of the light-curve shows little or no development from Oct. 1980 (Fig. 1) through Oct. 1981 and to Jan. 1983 (see Fig. 4 for the latter two dates). Further inspection of Fig. 4 shows that, between Jan. 1983 and Dec. 1983, a phase shift $\sim 0.2$ occurs, with the new form being maintained to Oct. 1984. Subsequently, in Nov. 1985, the light-curve presented a fairly nondescript structure with an amplitude less than 0 . 1 .

After a 10 year monitoring gap, the light-curve of Oct. 1995 is hardly distinguishable. However, in Oct. 1996 it appears to be quite sinusoidal with amplitude just greater than 0.1 and, with the selected period of 0.23540 , is in phase with the first reported curves of 1980. Three months later (Jan. 1997) the variation is radically different with a more spiky structure, this still being apparent in Oct. 1997. One year later (Oct. 1998), the light-curve has returned to have the characteristics of the original one recorded by vLAM. A further year on, a large change has occurred in terms of the shape and phase. Finally, in Mar. 2001, the variations have returned to a nondescript mode. It is very obvious that during the 20 year time interval, the spot structure on the star has suffered substantial changes. However, with respect to a period of 0.23540 , it may be noted that the curves of Oct. 1980, Oct. 1981, Jan. 1983, Oct. 1996 and Oct. 1998 are similar and, more importantly, in phase.

A question that might be raised is whether these latter noted light-curves are generated by near-identical spot dispositions re-establishing themselves around the same stellar longitudes, or whether the apparent phase matching just happens to occur as a result of fundamentally different spot distributions. If the first case applies, possible periods of $0.235400,0.235392$ 

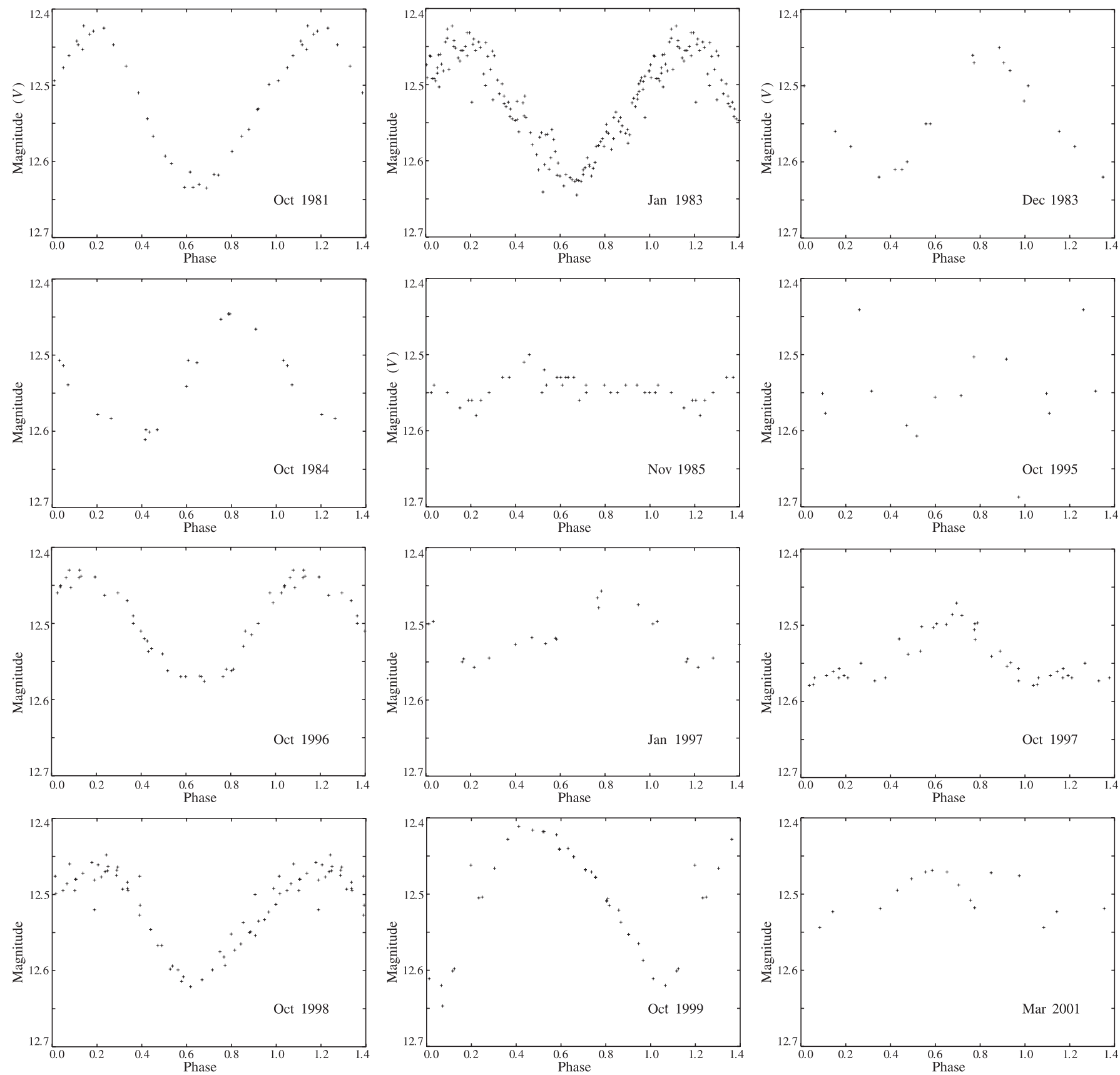

Fig. 4. The behaviour of the data, indicating the forms of the light-curves for HII 1883, based on the ephemeris HJD $=2444500.0+0.23540$, are shown for 12 observing seasons. Those measurements made in the standard system are labelled as $V$; for Oct. 1984, the observations were obtained as differential $V$ values; for Jan. 1983, the data were obtained as differential unfiltered photoelectric measurements; all other observations were made differentially using CCD small field imaging. Because the measurements originate from a range of instruments, there are differences in the effective wavelength from season to season. For those data comprising differential values, the magnitudes have been adjusted with arbitrary offset so that the curves are plotted within a matching range.

or 0.235408 emerge, the choice being dependent on the assumed number of intervening cycles, $N$, within the time base, but each carrying an individual uncertainty of \pm 0.000001 , this value appropriate in terms of the 20 year time base.

In order to promote the discussion of this theme further, some physical mechanism to reestablish spot distributions locked to some particular stellar longitudes would be required. It might be speculated, for example, that the star possesses a variable magnetic field with a dipolar component inclined to the rotational axis. At times when the field strength is high, features caused by the local magnetic pressure at the magnetic poles would dominate the photometric behaviour. It is noteworthy that Young et al. (1990) suggested the presence of two extended high latitude spots, separated in longitude by $180^{\circ}$ to explain the development in the light-curve of GL 890. Without alternative evidence for the preferred light-curve form being linked in phase by some particular spot distribution and, without any theoretical basis whereby BY Dra stars in general are 

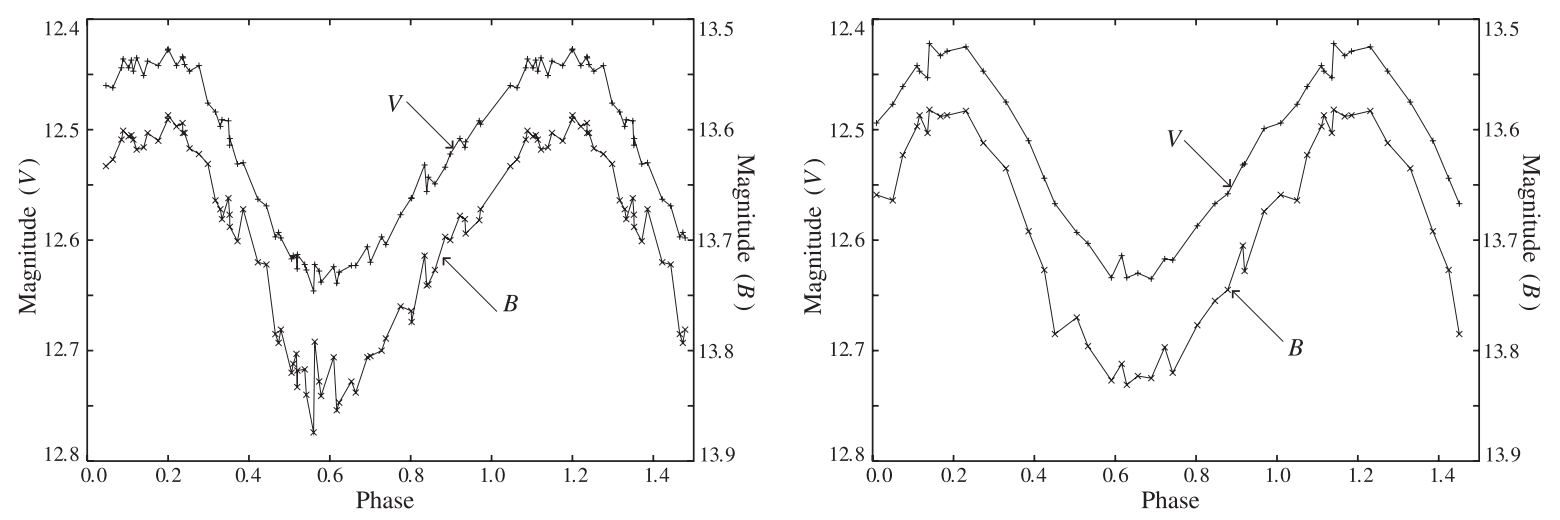

Fig. 5. The $B, V$ data of vLAM are phased on the ephemeris of HJD $=2444500.0+0.23540$. The left-hand plot is for Oct. 1980 and the right-hand plot is for Oct. 1981, with different scales assigned to $B$ and $V$ to allow their display within the same diagram. Successive phased points are linked to highlight the coherence of the small structures appearing on the underlying light-curves, the fluctuations being larger in the $B$-band than in $V$. The colour index change between maximum and minimum light is clearly seen in the $B-V$ difference at these phases.

likely to behave as magnetic oblique rotators, the proposition is not pursued. None of the three possible periods given above, carrying an additional decimal place are promoted as improvement to SDA's value of 0.23540 but they should be kept in mind in respect of any further photometric studies of HII 1883.

\section{Short term fluctuations}

All the measurements of HII 1883 tend to be made over concentrated spells, these being separated by intervals of the order of a year. Generally each observational run covers some 50 rotational cycles and a mean underlying light-curve is established which maintains its form after intervals of several thousand cycles. Inspection of the data from any one observing season folded on the rotational period shows the presence of noise. Whether this results from small intrinsic fluctuations in the star or is the result of experimental noise cannot be resolved from the existing data as the observational schemes were not especially configured for such an exercise. The following comments, however, may be made in terms of the apparent noise on the light-curve from cycle to cycle.

Inspection of the phased data plots for the $B$ and $V$-band measurements of vLAM in Oct. 1980 and Oct. 1981 (see Fig. 5) reveal "spiky" fluctuations when the plotted points are linked in sequence. The spikes display a correlated behaviour and relative to an underlying smooth light-curve, the departures tend to show as brightness enhancements, the $B$-band fluctuations being slightly larger than those for $V$. The spikes do not, however, represent short term fluctuations within a single cycle but are a result of the phase-folding process. Examination reveals that, over some sections of the light-curve, the data have phase overlap but with small brightness level changes from night-to-night. As the phase progresses in the plot, jumps from a point of one night to another and back again make the underlying light-curve appear spiky. In Fig. 6 the $B$-band data points for Oct. 1980 are redrawn with the effects of short term level changes from one cycle to another made apparent. This is particular evident within the construction of the light minimum. For example, the measurements made on the night of JD [2444]546.0+ run through with a

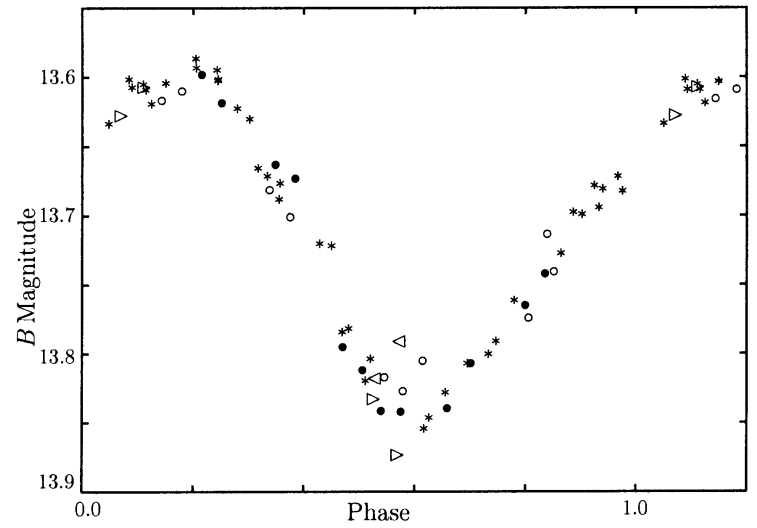

Fig. 6. The $B$-band measurements from four nights of the Oct. 1980 run are highlighted by symbols: nights with JDs of [244 4]546.0+, $553.0+, 559.0+$ and 571.0+ are plotted as $\bullet, \circ, \triangleleft$ and $\triangleright$ respectively; all other measurements appear as $*$. Note the difference in level for JD 546.0+ (•) and 553.0+ (०) through the light minimum. Also at the minimum, the sample measurements from JD 559.0+( $\triangleleft)$ appear to have higher brightness relative to JD $571.0+(\triangleright)$ by $\sim 0.1$.

minimum value $\sim 13$ m. 850 , whereas the minimum for JD 553.0+ is $\sim 0$. 025 brighter. Also at light minimum, the measurements of JD 559.0+ display significantly higher brightness relative to JD 571.0+. Around the minimum, the spread in brightness values over the observing window of some 30 days is $\sim 0 \mathrm{~m} 1$, such differences being in excess of the noise estimated from the general quality of the photometry. It may also be noted that if the correlation is investigated using sections of the $B, V$ data as plotted in Fig. 2, the coefficients are better defined around light maximum rather than at the minimum; a similar disparity occurs for the Oct. 1981 data. These indicators could be interpreted by the minimum corresponding to the presentation of the more disturbed hemisphere with the spots being "noisy" on time scales of a few stellar rotations.

The measurements of SDA(I) are plotted in Fig. 7 and show an underlying light-curve essentially the same as obtained by vLAM. Its inspection shows that the behaviour is not exactly repetitive on the three nights of observation. The measurements of JD 374.0+ appear slightly brighter on the descending slope 


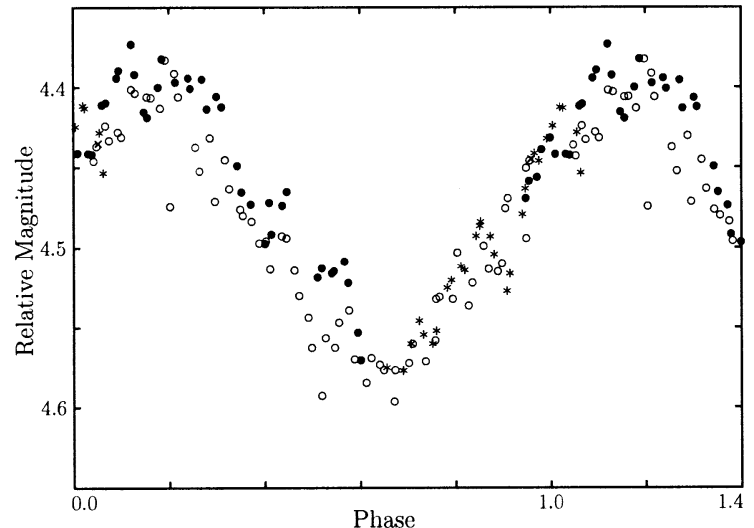

Fig. 7. The phased data of SDA(I), corresponding to unfiltered differential magnitude measurements between HII 1883 and comparison star, are plotted according to the ephemeris of HJD $=2444500.0+$ 0 d23540. The symbols $*, \circ$ and $\bullet$ correspond to the nights with JDs of [244 5]348.0+, 354.0+ and 374.0+, respectively. It may be noted that the descending slope of the light-curve on the night of $374.0+$ is consistently brighter than on $354.0+$.

relative to the night of JD $354.0+$ with a brightness bump near to light minimum. At face value, Fig. 7 suggests there are small changes over intervals $\sim 20$ days as though the disturbances giving rise to a particular form of photometric variation drift in position or intensity over a few tens of stellar rotations.

It is, of course, appreciated that the apparent night-to-night changes in the levels of parts of the light-curve could be engendered by instrumental effects and/or changes in the sky conditions affecting the data reductions. The fact that the levels in vLAM's $B$ and $V$ values are coherent might be explainable by noting that the photometer provides simultaneous colour measurements, with the possibility, that over and above the noise associated with photon counting statistics, any instrumental/data reduction noise affecting the colour channels is subject to coherent influences. Further photometry is required to investigate the issue of cycle-to-cycle variations, particularly at light minimum, with programmes designed to pay particular attention to measurement stability.

The apparent small photometric drifts, if intrinsic to the star, have parallels to the behaviour of other stars. The larger changes in $B$ with respect to $V$ are symptomatic of microflaring as reported for LQ Hya by Cutispoto (1991). The apparent level changes in the light minimum have similarities to the behaviour of AB Dor. According to observations by Kürster et al. (1994), the general light-curve shape for this star was maintained for 2 months but with significant deepening of the minimum; small changes to the rising and falling slopes were also noted. Over the three night monitoring of GL 890 by Young et al. (1990), the magnitudes within the light minimum display a spread greater than on other parts of the light-curve, again suggesting "flickering" at this phase.

\section{4. $\mathrm{H} \alpha$ variations}

The $\mathrm{H} \alpha$ emission line of HII 1883, surprisingly strong for a K-type star, was monitored over a single complete cycle by Marcy et al. (1985). The profile revealed complicated variations of structure with the velocity of the $\mathrm{H} \alpha$ centroid displaying a near-sinusoidal variation; there were also hints of small RV-curve variations occurring from one cycle to the next. No attempt was made by Marcy et al. (1985) to relate their $\mathrm{H} \alpha$ measurements to those of the continuum light-curve.

Phasing the $\mathrm{H} \alpha \mathrm{RV}$ values on the ephemeris of $H J D=2444500.0+0.23540$ shows that the times for zero velocity shift (see Fig. 4 of Marcy et al. 1985) occur at maximum and minimum brightness with respect to the nearidentical light-curves of Dec. 1983/Oct. 1984 (see Fig. 4), these recorded before and after the RV data (Jan. 1984). The forms of the asymmetries, relative to a pure sine wave, of the $\mathrm{H} \alpha \mathrm{RV}$ and continuum photometric curves also match. Thus, the stellar surface features giving rise to the light-curve appear to be linked to the $\mathrm{H} \alpha$ emission line $\mathrm{RV}$ variations. When the features are at the centre of the projected stellar disk and moving across the line of sight, the core of the $\mathrm{H} \alpha$ emission displays zero radial velocity, as might be expected. The equivalent width of the emission was very noisy from one cycle to another, but over the monitored full period, times of maximum and minimum $\mathrm{H} \alpha$ equivalent widths match those of maximum and minimum continuum brightness.

Thus, there appears to be a relationship between the disposition of the features giving rise to the continuum photometric behaviour and the lumped $\mathrm{H} \alpha$ emission. Each recorded $\mathrm{H} \alpha$ profile displays substantial structure, however, as though it might be generated from many zones within the stellar chromosphere. An important observational exercise for the future would be to monitor these structures to confirm their coherent progress across the emission line. This has been done in the time-resolved $\mathrm{H} \alpha$ study of AB Dor by Collier Cameron \& Robinson (1989a,b) with the recording of several absorption features passing through the emission profile.

\section{A simple multi-cell model}

Following their observations, SDA applied the model of Dorren et al. (1985) to the HII 1883 light-curve as it appeared prior to 1985 . This model uses two or more spots whose radii, locations and temperatures can be varied. Because of the noted high $v \sin i$, as determined by spectrometry (see, Jones et al. 1996), the value of $i$ must be close to $90^{\circ}$ and SDA arbitrarily set it at $75^{\circ}$. With such a high value, a single spot would provide a light-curve with flat sections at times when the feature is on the far stellar face and obscured. To produce nearsinusoidal light-curves, incorporation of at least two spots is required, this being the strategy of SDA. From the structures appearing in the $\mathrm{H} \alpha$ emission, a question arises as to the morphologies of light-curves generated by the presence of many photospheric spots. This is pursued below in respect of stars with a high value of inclination, the disturbances appearing at low latitude. Effects on the resulting light-curve are also explored by allowing the strength of one of the spot elements to vary in brightness. The model does not rule out the possibility of only two spots being present as in the model of SDA but, if nothing else, highlights the inversion problems associated with relating photometric measurements to particular underlying spot distributions. 


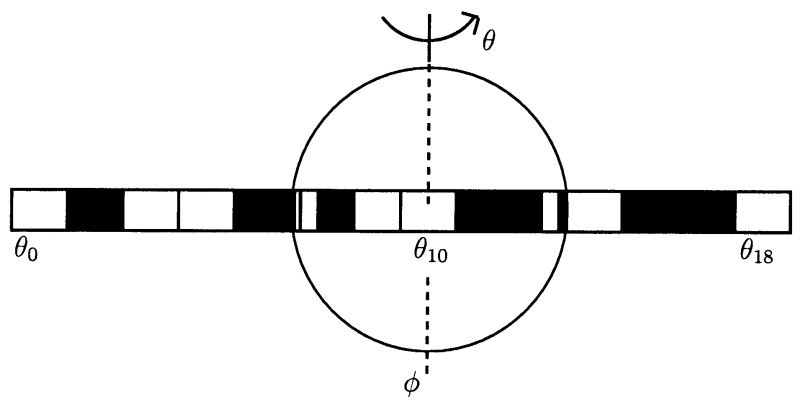

Fig. 8. The equatorial strip of a model star viewed at inclination $i=$ $90^{\circ}$ is divided into 18 equal arc segments containing features which are either "switched" on or off, their distribution mimicking a random mottled effect. Segments towards the stellar limb display foreshortening. The unseen segments on the rear face are displayed on either side of the disk, their effects influencing the light-curve as stellar rotation carries them onto the observed hemisphere. The example above has selected $\theta_{2}, \theta_{5}, \theta_{8}, \theta_{11}, \theta_{12}, \theta_{14}, \theta_{16}, \theta_{17}$ as carrying dark features, with segment $\theta_{10}$ on the stellar meridian.

For simplicity the equatorial strip is divided into an even number, $N$, of equally-sized segments, each with arc $2 \pi / N$ radians along the equator. It is assumed here that the features are dark spots, although the general outcomes in terms of the generated light-curves would be the same if bright features were to be considered. The energy flow from each segment may be defined as either $B_{\mathrm{p}}$ or $B_{\mathrm{s}}$, depending on whether the segment is respectively undisturbed or corresponds to a darkened feature.

Suppose the segments are described in terms of longitude around the stellar equator and identified as $\theta_{1}, \theta_{2}, \theta_{3}, \ldots, \theta_{N}$, with $\theta_{1}$ acting as reference for zero longitude and providing the phase fiduciary of the light-curve. Suppose also that $\phi$ defines the fixed meridian of the star as seen by the observer such that when $\theta_{1}-\phi=0$, zero longitude runs through the centre of the projected stellar disk. When the centre of any segment is set at $\phi$, the observer receives radiation from it and the adjacent $N / 4$ equatorial segments either side of the meridian, their contributions being affected by foreshortening according to their position, plus the radiation from the polar regions either side of the disturbed equatorial belt. Effects of the other $N / 2$ sectors are excluded as they are temporarily on the reverse hemisphere.

By assuming that the contribution of each segment is affected simply by foreshortening, with other complications such as limb darkening ignored, the photometric signal when the $i$ th segment is on the meridian may therefore be written simply as

$I\left(\theta_{i}\right) \Longrightarrow K\left[B+\sum_{j=i-N / 4}^{i+N / 4} X_{j} \cos \left(\theta_{j}-\phi\right)\right]$,

where $B$ represents the contributing brightness of the undisturbed regions either side of the equator and $X_{j}$ takes values of either $B_{\mathrm{p}}$ or $B_{\mathrm{s}}$ according to the setup of the particular run of the model; the constant $K$ depends on the size of the star and on its distance from the Earth.

For a given run of the model, consecutive segments are set at random as being either active or undisturbed, so providing

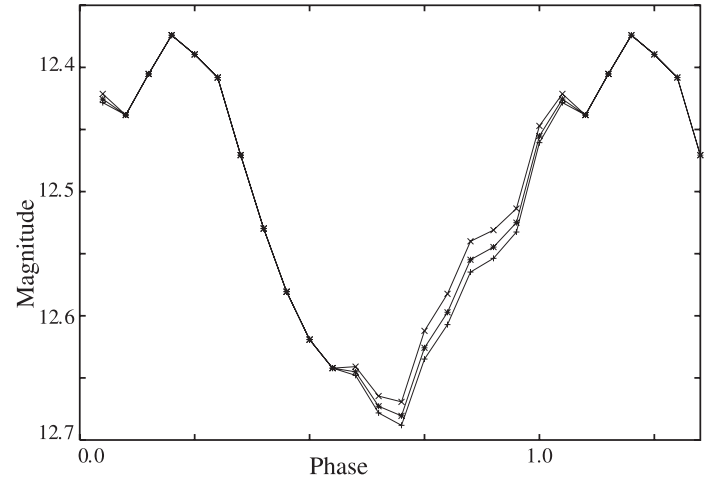

Fig. 9. An example light-curve based on 20 cells selected at random as being active/inactive has been scaled to provide an approximate match to the behaviour of HII 1883. Comparison with Fig. 1 illustrates the similarities of engendered and real light-curves, with steeper fall in brightness relative to the rise towards maximum light. One of the active cells was made progressively darker, so deepening the light minimum and affecting the rising slope.

a mottled pattern of the kind as depicted in Fig. 8. The form of the light-curve from the generated pattern may then be developed by letting $K=1$, setting $B$ to some arbitrary value and letting $B_{\mathrm{p}}$ be some fraction of $B$, say $1 / 100$ with $B_{\mathrm{s}}$ being slightly smaller, say $1 / 101$. Values of $I\left(\theta_{i}\right)$ may be obtained producing $N$ points on the light-curve corresponding to the times when each segment in turn is centrally on the stellar meridian.

The generated light-curves generally display nearsinusoidal variations with amplitude depending on the contributions of $B_{\mathrm{p}}$ and $B_{\mathrm{s}}$ relative to $B$, on the differences between the levels of $B_{\mathrm{p}}$ and $B_{\mathrm{s}}$ and on the value of $N$. This may be interpreted simply in terms of the sizes of the spots (e.g., their spread in latitude), and on the temperature differences between the undisturbed photosphere and the darkened regions. In addition, the larger the value of $N$, the more the light-curve is smoothed out and reduced in amplitude relative to the undisturbed level.

An example of a generated light-curve with $N=20$ and scaled to a magnitude for comparison with HII 1883 (see Fig. 1) is displayed in Fig. 9. The 20 photometric points provide an unsmoothed light-curve, this resulting from the integer nature of the cell pattern and from not allowing for their smooth progression at ingress and egress at the stellar limb. It can be seen, however, that there are strong similarities between the engendered and real light-curve, with an asymmetry apparent in the steeper fall in brightness relative to the rise towards maximum light. It will be remembered that the model uses equally-sized cells with the active ones made darker by the same degree. Similar forms of light-curve ensue if the segments are made more random in size and in their degree of darkening.

To mimic the effect of a cell fluctuating in intensity, one of them was selected and allowed to grow darker from one rotational cycle to the next. In the example of a model run (see Fig. 9) the basic shape of the light-curve is a good match to the recurring one of HII 1883 while its development mimics that of AB Dor as monitored by Kürster et al. (1994). It can be seen that on the half of the cycle when this developing spot appears on the visible hemisphere, the basic light-curve form is 
maintained but the light minimum deepens and the rising slope is affected.

The behaviour of light-curves generated by the multi-spot model is not particularly sensitive to the latitude of the active belt. The general conclusion agrees with an alternative analysis by Clarke (2003). According to this work, the effect of a spot which is obscured during a large fraction of the rotational cycle contributes a photometric signal dominated by the first harmonic (三 rotational period) and second harmonic. Effects of additional spots of differing "strength" may be synthesised by co-adding their signal Fourier amplitudes, dominated by first and second harmonic terms, according to their phases, these being determined by the spots' locations. Thus BY Dra stars tend to display light-curves of similar form. Analyses of their light-curves are obviously incapable of producing unique descriptions of the underlying spot distribution.

\section{Conclusion}

Various photometric data of HII 1883 have been assessed collectively to see if variations of the period are detectable as a result of latitude migration of surface features in association with differential rotation or as a result of evolutionary spin-down. The null result indicates that the accuracy of synoptic period determinations require significant improvement to detect these effects which, no doubt, are present.

HII 1883 exhibits light-curves which may persist for thousands of cycles. Occasional changes in phase and curve shape make it impossible to simply extend the time base and increase the accuracy of the determination of the period. It is noted, however, that one particular light-curve seems to recur after substantial time intervals. Further, regular photometric monitoring is required to check this tendency with respect to phase coherence. If it is confirmed, it provides a basis for improving the knowledge of the stellar rotational period and, more importantly, it has consequences on the nature of the star's structure with the spots recurring at a particular stellar longitude.

In future monitoring programmes, it is also important undertake a more focussed observational strategy to check on the suspected light-curve variability on time scales of a few rotational cycles. Such results have bearing on physical processes associated with the active nature of the disturbances. Subject to the availability of a sufficiently large telescope aperture, contemporaneous high resolution observations of the $\mathrm{H} \alpha$ emission, with Doppler imaging and magnetographic studies using other lines, would expand the understanding of the nature and disposition of the active regions on HII 1883.
Acknowledgements. My sincere thanks are due to Dr. Floor van Leeuwen for providing an electronic listing of the data which appeared in vLAM (1987) and to Dr Dana Backman for arranging transmission of data files containing the more recent CCD photometry of HII 1883. Thanks for the assembly of the latter material are also due to Dr Laurence Marschall (Gettysburg College) and to Candace Grand Pré (Franklin and Marshall College). Finally, the author wishes to acknowledge the anonymous referee who helped to improve the form of the paper.

\section{References}

Alphenaar, P., \& van Leeuwen, F. 1981, IBVS, 1957

Barnes, S. A., Sofia, S., Prosser, C. F., \& Stauffer, J. R. 1999, ApJ, 516,263

Clarke, D. 2002, A\&A, 386, 763

Clarke, D. 2003, A\&A, 407, 1029

Collier Cameron, A., \& Robinson, R. D. 1989a, MNRAS, 236, 57

Collier Cameron, A., \& Robinson, R. D. 1989b, MNRAS, 238, 657

Cutispoto, G. 1991, A\&AS, 89, 435

Donati, J.-F., \& Collier Cameron, A. 1997, MNRAS, 291, 1

Dorren, J. D., Siah, M. J., Guinan, E. F., \& McCook, G. P. 1985, AJ, 86,572

Endal, A. S., \& Sofia, S. 1981, ApJ, 243, 625

Innes, J. L., Thompson, K., \& Coates, D. W. 1986, MNRAS, 223, 183

Jones, B. F., Shetrone, M., Fischer, D., et al. 1996, AJ, 112,186

Krishnamurthi, A., Terndrup, D. M., Pinsonneault, M. H., et al. 1998, ApJ, 493, 914

Kürster, M., Schmitt, J. H. M. M., \& Cutispoto, G. 1994, A\&A, 289, 899

Marcy, G. W., Duncan, D. K., \& Cohen, R. D. 1985, ApJ, 288, 259

Hussain, G. A. J., Unruh, Y. C., \& Collier Cameron, A. 1997, MNRAS, 288, 343

Meys, J. J. M., Alphenaar, P., \& van Leeuwen, F. 1982, IBVS, 2115

Radick, R. R., Thompson, D. T., Lockwood, G. W., et al. 1987, ApJ, 321,459

Stauffer, J. R. 1984, ApJ, 280, 189

Stauffer, J. R., Dorren, J. D., \& Africano, J. L. 1986, AJ, 91, 1443

Stauffer, J. R., Hartmann, L., Soderblom, D. R., \& Burnham, N. 1984, ApJ, 280, 202

Stout-Batalha, N. M., \& Vogt, S. S. 1999, ApJS, 123, 251

Strassmeier, K. G., Rice, J. B., Wehlau, W. H., Hill, G. M., \& Matthews, J. M. 1993, A\&A, 268, 671

van Leeuwen, F., \& Alphenaar, P. 1982, ESO Messenger, 28, 15

van Leeuwen, F., Alphenaar, P., \& Meys, J. M. M. 1987, A\&AS, 67, 483

Vogt, S. S. 1981, ApJ, 250, 327

Young, A., Skumanich, A., MacGregor, K. B., \& Temple, S. 1990, ApJ, 349, 608 